\title{
Prevalence of Methicillin-Resistant Staphylococcus Aureus (MRSA) infection in patients with Cystic Fibrosis: a meta-analysis
}

\section{Sagad Omer Obeid Mohamed ( $\nabla$ s.oom123@yahoo.com )}

University of Khartoum Faculty of Medicine

Almigdad H. M. Ali

University of Khartoum Faculty of Medicine

Abazr A. H. Ibrahim

University of Khartoum Faculty of Medicine

Mahmoud Elnil

University of Khartoum Faculty of Medicine

Almutasim B. E. Elhassan

University of Khartoum Faculty of Medicine

Mohammed Suliman Tawer Salman

University of Khartoum Faculty of Medicine

Ibrahim H. E. Elkhidir

University of Khartoum Faculty of Medicine

\section{Osman Omer Ali}

University of Khartoum Faculty of Medicine

Mazin A.M. Elhassan

University of Khartoum Faculty of Medicine

Mohamed Elata Hassan Elbathani

University of Khartoum Faculty of Medicine

Research article

Keywords: Cystic fibrosis, MRSA, Prevalence, Meta-analysis.

Posted Date: July 18th, 2019

DOl: https://doi.org/10.21203/rs.2.11668/v1

License: (c) (1) This work is licensed under a Creative Commons Attribution 4.0 International License.

Read Full License 


\section{Abstract}

Background Methicillin-resistant staphylococcus aureus (MRSA) infection is increasingly being reported among patients with cystic fibrosis (CF) and contributes to pulmonary morbidity in CF, with poorer prognosis. The aim of this study was to assess the prevalence of MRSA infection in patients with CF. Methods We conducted this study according to the Preferred Reporting Items for Systematic Reviews and Meta-Analyses (PRISMA) guidelines. Databases of MEDLINE/PubMed, WHO-Virtual Health Library (VHL), ScienceDirect, Google Scholar and OpenGrey were searched to recruit the relevant articles. Pooled prevalence with the corresponding $95 \%$ confidence interval $(\mathrm{Cl})$ was calculated using OpenMeta Analyst software, and heterogeneity among studies was estimated using the 12 statistics. Results According to our inclusion criteria, 27 studies included a total of 47,413 patients were analyzed. The pooled prevalence of MRSA in patients with CF was $15.2 \%$ (95\% Cl 9.70\%- 20.7\%). Subgroup analyses and meta-regression showed that the prevalence of MRSA in patients with CF was significantly associated with different geographical areas $(P<0.001)$, data collection method $(P<0.001)$, sample obtaining source $(P<0.001)$, and study year $(P=0.006)$. Conclusions prevalence of MRSA infection is increasing in patients with $C F$. the results of this study could provide a reference for further controlling transmission and the management of patients with CF. Healthcare providers need to be aware of the clinically important association between MRSA infection and CF to ensure effective management.

\section{Introduction}

Cystic fibrosis (CF) is an autosomal recessive multisystem genetic disease, and one of the commonest life-threatening disorders caused by mutations in the $\mathrm{CF}$ transmembrane conductance regulator (CFTR) gene on chromosome 7, leading to production of thick secretions that predispose to chronic pulmonary infections, digestive tract disorders, as well as other systems impairment [1-2]. Most of patients with CF develop respiratory failure brought on by recurrent and chronic bacterial infection leading to persistent lung infections \& obstructive lung disease, which is the most common cause of death in this population [3]. Respiratory tract colonization by these pathogenic microorganisms in patients with $\mathrm{CF}$ represents a serious health problem and it is considered a major cause of morbidity and mortality [4-5].

Patients with cystic fibrosis are susceptible to respiratory tract colonization and infections by different microorganisms due to their thick viscid secretions and impaired mucociliary clearance mechanism [6]. Classic organisms isolated from patients with cystic fibrosis include methicillin-sensitive staphylococcus aureus (MSSA), methicillin-resistant staphylococcus aureus (MRSA), Pseudomonas aeruginosa, and Burkholderia capacia [3]. MRSA is considered among pathogens of the highest prevalence, and a significant cause of increased morbidity and mortality among patients with CF [7]. Methicillin resistance seriously compromises the choice of antibiotic treatment in these patients [8]. Recent epidemiologic studies have evaluated the role of MRSA as contributor to clinical outcomes in patients with CF, and a risk factor for failure to recover lung function after an acute pulmonary exacerbation [9-10]. 
Studies have demonstrated that patients with MRSA detected in their sputum cultures and bronchial secretions had more hospital admissions per year as well as increased length of hospitalization and impaired pulmonary function tests than in patients without MRSA even after hazard ratios adjustment. Thus, it greatly impairs the quality of life of the affected patients. Furthermore, it has been linked with poor survival [11-13].

Several studies assessed the prevalence of MRSA in patients with CF and reported a progressive increase in the prevalence. However, these studies remain inconsistent with wide variation in the data obtained from these studies, and to the best of our knowledge, there is no meta-analysis of existing contemporary evidence on the prevalence of MRSA in patients with CF. The systematic measurements of the prevalence of MRSA will show the magnitude of this problem and the results of this study can provide a comprehensive view of MRSA prevalence in this susceptible population and may contribute to its control and management.

\section{Methods}

Search strategy and inclusion criteria:

The methodology was developed from the Preferred Reporting Items for Systematic Reviews and MetaAnalyses (PRISMA) statement [14]. Medline/PubMed, WHO-Virtual Health Library (VHL), Google Scholar, ScienceDirect and OpenGrey were searched for all studies on association between MRSA infection and CF. We performed a systematic literature search of the database up to February 2019 using the search terms "methicillin-resistant staphylococcus aureus", "MRSA", "cystic fibrosis", and "CF" to ensure maximal coverage of possible literature.

The criteria for paper inclusion were any study published in English that presented the prevalence estimates of MRSA in patients with CF or sufficient data for estimation. If two or more studies shared the same patient population, the study with more complete data or a larger sample size was included to avoid duplication. Infection was defined as the presence of this microorganism as detected by isolation from the specimens obtained from patients with $\mathrm{CF}$, weather it was described as intermittent or persistent colonization in the included studies. Study exclusion criteria included the following: case reports, case series, editorial letters, reviews, abstracts, and studies lacking the data of interest.

The titles and abstracts of all papers retrieved from this search were screened for potential inclusion in this review. Then, potentially relevant studies were reviewed for inclusion according to the defined eligibility criteria. Any disparity was resolved by discussion and consensus. Quality of the studies was assessed using a quality assessment tool for prevalence studies suggested by Hoy et al, which is a tool that addresses internal and external validity issues of prevalence studies based on combined criteria [15]. Data extraction form was developed to extract the relevant information, and the following data were extracted: authors, study region, data collection method, samples' sources, year of publication, gender, patients' age group, number of patients with CF and number of patients with MRSA. 
Statistical analysis:

Pooled prevalence from the random effects model with the corresponding 95\% confidence interval $(\mathrm{Cl})$ was calculated using OpenMeta Analyst software version 10.10 for analysis [16]. Heterogeneity among studies was estimated using the $\mathrm{I}^{2}$ statistics and publication bias was estimated by visual examination of the funnel plot. We detected a substantial level of heterogeneity $\left(I^{2}=99.7 \%\right)$ in estimated effect size between the included studies. Therefore, factors associated with the studies themselves (study area, data collection methods, sampling source, publication year and sample size) may have a moderating effect on the overall results. We therefore conducted subgroup analyses and meta-regression to determine the extent to which these variables moderated the overall results. Chi-square $\left(X^{2}\right)$ test was used to assess the differences between the categorical subgroups and the significance level was set at 0.05 .

\section{Results}

The schematic flow of study identification and selection process is presented in (Figure 1). The initial search retrieved records for 750 published articles. Full texts of 50 studies were screened and 23 studies of which were subsequently omitted because of low quality and insufficient data to estimate the outcomes of interest. Lastly, a total of 27 eligible studies published from 1988 to 2019 which met the eligibility for data extraction and analyses were used for qualitative and quantitative syntheses; 15 studies from Europe [4, 8, 17-29], 9 studies from the Americas [5, 30-37], 2 studies from Asia [38-39] and 1 study from Africa [40]. Overall, this analysis included a total of 47,413 patients. Most of the reviewed studies included all of the age groups (children and adult patients), whereas 7 studies were conducted exclusively among children. CF patients in these studies were diagnosed by positive sweat tests and/or presence of the CFTR mutations. The main characteristics of these included studies are shown in (Table 1 in the Supplementary Files).

Meta-analysis for the included studies showed that the prevalence of MRSA in this population from the random effects model was $15.2 \%$ (95\% Cl $9.70 \%-20.7 \%$ ) (Figure2). A slight publication bias was detected based on visual examination of the funnel plot (figure 3 ). In subgroup analysis, the pooled prevalence of MRSA infection among patients with CF was 8.70\% (95\% $\mathrm{Cl} 6.40 \%-10.9 \%)$ in Europe, $19.0 \%(95 \% \mathrm{Cl} 6.20 \%-13.8 \%)$ in the Americas, and $27.5 \%(95 \% \mathrm{Cl}-0.026 \%-0.576 \%)$ in Asia (Figure 1$)$. There was a statistical difference in prevalence between different geographical areas $\left(X^{2}=140.4, P \bigotimes\right.$ $0.001)$, Specimen obtaining sources $\left(X^{2}=84.42, P \llbracket 0.001\right)$, and data collection methods $\left(X^{2}=6373.6, P \square\right.$ 0.001) (Table 2 in the Supplementary Files). Furthermore, meta-regression analyses were done to analyze whether the continuous variables (sample size and publication year) affected the heterogeneity among studies in this meta-analysis. The results showed that publication year was correlated with the outcome $(P=0.006)$, and sample size had no moderating effects on the outcome of this analysis $(P=0.936)$ (Figure 4 A-B).

\section{Discussion}


During the disease course, patients with CF are susceptible to infection with several microorganisms including MRSA, leading to frequent episodes of debilitating inflammatory exacerbations and progressive lung damage, influencing the quality of life and life expectancy [41]. Most of the included studies in this meta-analysis assessed the prevalence of MRSA infection in the European CF population, which is consistent with the distribution areas of patients with $\mathrm{CF}$, which is found predominantly in Caucasian populations of European ancestry [3]. In this meta-analysis, we have an updated estimate of the global prevalence of MRSA colonization in CF population. Existing evidence from the reviewed studies showed that $15.2 \%$ of CF patients were infected to the MRSA.

The discrepancy between prevalence rates reported from the reviewed studies might be attributable to several factors. In this meta-analysis, we have found that the prevalence of MRSA showed regional epidemiological differences. This finding was also reported by previous review done Christopher $\mathrm{H}$. Goss et al [9]. While rates of MRSA have been reported to be considerably lower in most European CF centres, the prevalence tends to be a little higher in other countries where there is overcrowded living condition and inadequate implementation of infection control measures.

MRSA poses a concern because of its rising prevalence and its rapidly growing antibiotic resistance attributed to its resilience and different molecular properties that facilitate the acquisition of different antibiotic resistance genes [42]. In this meta-analysis, we found that the prevalence of MRSA infection has climbed up over years. This increase is in line with previous reviews done by LiPuma, Kahl and Jennings et al $[3,10,43]$. They reported that MRSA infection among CF population has demonstrated a notable increase in prevalence over the last decades. Analysis of data from the Cystic Fibrosis Foundation Patient Registry in the United States indicates that the prevalence of MRSA infection in CF patients increased steadily during the last years [3]. Also, this observation has been noted in a reference centre for the treatment of CF patients in Buenos Aires, Argentina, where the prevalence of MRSA has climbed from 23\% in 1995 up to 32\% in 2011 [34].

Sputum specimen is a common and readily available method for clinical evaluation of the lung microorganisms in patients with CF. It is less sensitive than other more invasive procedures such as bronchoalveolar lavage, but it remains an accurate indicator of lower airway microbiology [44]. Most of the included studies data came from the results of sputum samples. This meta-analysis showed that the colonization prevalence of MRSA from sputum culture alone was lower than that from specimens obtained from sputum with other more invasive sampling.

Other possible risk factors of MRSA infection in patients with CF have been assessed by a few of the included studies. Some of these studies had demonstrated that older age [22], MRSA detection in family members [17], occupational exposure [38], frequent hospitalization [21, 34, 37], CF-related diabetes [34], pancreatic insufficiency [21], pre-existing bronchiectasis [21], and Pseudomonas aeruginosa co-infection $[17,31]$ could be implicated in the increasing burden of MRSA infection in patients with CF. 
Limitations: The findings of this study need to be considered in the context of some limitations. The inclusion of studies published only in English may compromise representativeness. As well, because of the lack of uniformity across studies, we did not assess the factors that possibly increase the risk of MRSA infection among patients with CF.

\section{Conclusion}

We have summarized data of several studies exploring the prevalence of MRSA among patients with CF in this study. MRSA infection occurs with increased frequency in patients with CF. the results of this study could have an important implication for further controlling transmission, and could provide a reference for the management of patients with CF. Healthcare providers need to be aware of the clinically important association between MRSA infection and CF to ensure effective management.

\section{Abbreviations}

MRSA: methicillin-resistant staphylococcus aureus; CF: cystic fibrosis

\section{Declarations}

- Ethical approval and consent to participate: not applicable

- Consent for publication: not applicable.

- Availability of data and material: The dataset generated during this study are available from the corresponding author on reasonable request.

- Competing interests: The authors declare that they have no competing interests.

- Funding: No fund.

- Authors' contribution: (SM) conceptualized the research idea and designed the study; (SM, AA and Al) undertook articles searching; (SM, ME, AE and MS) undertook data extraction and analysis; All authors interpreted the results and drafted the manuscript. All authors revised and approved the final manuscript.

- Acknowledgment: The authors would like to acknowledge Abdalla Omer for helping us in data extraction.

\section{References}

1- Misbahuddin M. Rafeeq, Hussam Aly Sayed Murad. Cystic fibrosis: current therapeutic targets and future approaches and J Transl Med. 2017; 15: 84. doi: 10.1186/s12967-017-1193-9 2- Susanne Naehrig, Cho-Ming Chao, Lutz Naehrlich. Cystic Fibrosis Diagnosis and Treatment Dtsch Arztebl Int. 2017 Aug; 114(33-34): 564-574. doi: 10.3238/arztebl.2017.0564 3- Lyczak JB, Cannon CL, Pie GB. Lung Infections Associated with Cystic Fibrosis. CLINICAL MICROBIOLOGY REVIEWS. 2002, p. 194-222 Vol. 15, No. 2. DOI: 10.1128/CMR.15.2.194-222.2002 4- Auxiliadora Molina, Rosa Del Campo, Luis Ma'iz, Marı́a-Isabel 
Morosini, Adelaida Lamas, Fernando Baquero, Rafael Canto' n. High prevalence in cystic fibrosis patients of multiresistant hospital-acquired methicillin-resistant Staphylococcus aureus ST228-SCCmecl capable of biofilm formation. Journal of Antimicrobial Chemotherapy (2008) 62, 961 - 967 doi:10.1093/jac/dkn302 5- Vilma Almeida Paixão, Tânia Fraga Barros, Clélia Maria C Mota, Tamy Fagundes Moreira, Maria Angélica Santana, Joice Neves Reis. Prevalence and antimicrobial susceptibility of respiratory pathogens in patients with cystic fibrosis. Braz J Infect Dis 2010; 14(4):406-409 6- Köck R, Becker K, Cookson B, van Gemert-Pijnen JE, Harbarth S, Kluytmans JA, Mielke M, Peters G, Skov RL, Struelens MJ, Tacconelli E. Methicillin-resistant Staphylococcus aureus (MRSA): burden of disease and control challenges in Europe. 7- LiPuma JJ. The Changing Microbial Epidemiology in Cystic Fibrosis. CLINICAL MICROBIOLOGY REVIEWS, Apr. 2010, p. 299-323 Vol. 23, No. 2 Doi:10.1128/CMR.00068-09 8S. R. Thomas, K. M. Gyi, H. Gaya, M. E. Hodson. Methicillin-resistant Staphylococcus aureus: impact at a national cystic fibrosis centre. journal of Hospital Infection ( 1998) 40: 203-209. 9- Goss CH, Muhlebach MS. Review: Staphylococcus aureus and MRSA in cystic fibrosis. Journal of Cystic Fibrosis 10 (2011) 298 - 306 10- Barbara C. Kahl. Impact of Staphylococcus aureus on the pathogenesis of chronic cystic fibrosis lung disease. International Journal of Medical Microbiology 300 (2010) 514-519. 11- Miall LS, McGinley NT, Brownlee KG, Conway SP. Methicillin resistant Staphylococcus aureus (MRSA) infection in cystic fibrosis. Archives of disease in childhood. 2001 Feb 1;84(2):160-2. 12- Dasenbrook EC, Checkley W, Merlo CA, Konstan MW, Lechtzin N, Boyle MP. Association between respiratory tract methicillin-resistant Staphylococcus aureus and survival in cystic fibrosis. Jama. 2010 Jun 16;303(23):2386-92. 13- Goodrich JS, Sutton-Shields TN, Kerr A, Wedd JP, Miller MB, Gilligan PH. Prevalence of community-associated methicillin-resistant Staphylococcus aureus in patients with cystic fibrosis. Journal of clinical microbiology. 2009 Apr 1;47(4):1231-3. 14- Liberati A, Altman DG, Tetzlaf J, Mulrow C, Gotzsche PC, loannidis JP, Clarke M, Devereaux PJ, Kleijnen J, Moher D. The PRISMA statement for reporting systematic reviews and meta-analyses of studies that evaluate health care interventions: explanation and elaboration. J Clin Epidemiol. 2009;62(10):e1-34. 15- Hoy D, Brooks P, Woolf A, et al. Assessing risk of bias in prevalence studies: modification of an existing tool and evidence of interrater agreement. J Clin Epidemiol 2012;65:934-9 16- OpenMetaAnalyst, Wallace BC, Dahabreh IJ, Trikalinos TA, Lau J, Trow P, Schmid $\mathrm{CH}$. Closing the gap between methodologists and end-users: $\mathrm{R}$ as a computational back-end. $J$ Stat Softw. 2012;49:5. 17- Matthias Kappler, Felicitas Nagel, Maria Feilcke, Carolin Kr €oner, Ingo Pawlita, e al. Eradication of Methicillin Resistant Staphylococcus aureus Detected for the First Time in Cystic Fibrosis: A Single Center Observational Study. Pediatric Pulmonology. DOI 10.1002/ppul.23519 18Dominique Hubert, et al. Association between Staphylococcus aureus alone or combined with Pseudomonas aeruginosa and $t$ he clinical condition of patients with cystic fibrosis. Journal of Cystic Fibrosis 12 (2013) 497 - 503. http://dx.doi.org/10.1016/j.jcf.2012.12.003 19- A. Solis, D. Brown, J. Hughes, H.K.F. van Saene. Methicillin-Resistant Staphylococcus aureus in Children With Cystic Fibrosis: An Eradication Protocol Pediatric Pulmonology. 36:189-195 (2003). 20- Giuseppe Valenza, Dennis Tappe, Doris Turnwald, Matthias Frosch, Corinne König, Helge Hebestreit, Marianne Abele-Horn. Prevalence and antimicrobial susceptibility of microorganisms isolated from sputa of patients with cystic fibrosis. Journal of Cystic Fibrosis 7 (2008) 123 -127. 21- E. Vanderhelst, L. De Meirleir, S. Verbanck, D. Piérard, W. Vincken, A. Malfroot. Prevalence and impact on FEV1 decline of chronic methicillin-resistant 
Staphylococcus aureus (MRSA) colonization in patients with Cystic Fibrosis A single-center, case control study of 165 patients. Journal of Cystic Fibrosis 11 (2012) 2 - 7 22- A. Vergison, O. Denis, A. Deplano, G. Casimir, G. Claeys, F. DeBaets, K. DeBoeck, N. Douat, et al. National survey of molecular epidemiology ofStaphylococcus aureus colonization in Belgian cystic fibrosis patients. Journal of Antimicrobial Chemotherapy (2007) 59, 893 - 899. doi:10.1093/jac/dkm037 23- Hector A, et al, Microbial colonization and lung function in adolescents with cystic fibrosis, J Cyst Fibros (2016), http://dx.doi.org/10.1016/j.jcf.2016.01.004 24- Pinar Yurdakul , Hamdiye Yesim Ocal, Dolunay Gulmez, Ebru Yalcin, Deniz Dogru. Predominance of hospital-associated MRSA among cystic fibrosis patients in a Turkish reference cystic fibrosis centre. Journal of Chemotherapy 2012; 24:4 25- Silvia Campana, Giovanni Taccetti, Novella Ravenni, Isabella Masi, Sandra Audino, Barbara Sisi, Teresa Repetto, Gerd Doring, Maurizio de Martino. Molecular epidemiology ofPseudomonas aeruginosa, Burkholderia cepaciacomplex and methicillin-resistant Staphylococcus aureus in a cystic fibrosis center. Journal of Cystic Fibrosis 3 (2004) 159 - 163 26- Dodémont M, et al. Emergence of livestock-associated MRSA isolated from cysticfibrosis patients: Result of a Belgian national survey, J Cyst Fibros (2018), https://doi.org/10.1016/ j.jcf.2018.04.008 27-Cox, D W, Kelly, C;Rush, R;O'Sullivan, N;Canny, G;Linnane, B. The impact of MRSA infection in the airways of children with cystic fibrosis; a case-control study. Ir Med J. 104 (10):305-8 28- Joana Fermeiro, Patrícia Reis, Susana Castanhinha, Luísa Pereira, Celeste Barreto. The impact of methicillin-resistant Staphylococcus aureus colonisation on paediatric cystic fibrosis patients' morbidity. Rev Port Pneumol 2010; 26 (4): 527-542 29- V. Cafiso, T. Bertuc cio, D. Spina, F. Campan ile, D. Bongiorno, M. San tagati, et al Methicillin resistance and vancomycin heteroresistance in Staphy lococcus aureus in cystic fibrosis patients. Eur J Clin Microbiol Infect Dis (2010) 29:1277- 1285 DOI 10.1007/s10096-010-1000-5 30- Bernard Boxerbaum, Michael R. Jacobs, Ronald L. Cechner. Prevalence and Significance of Methicillin-Resistant Staphylococcus aureus in Patients With Cystic Fibrosis. Pediatric Pulmonology 4:159-163 (1988). 31- Maliniak ML, et al, A longitudinal analysis of chronic MRSA and Pseudomonas aeruginosaco-infection in cysticfibrosis: A single-center study, J Cyst Fibros (2015), http://dx.doi.org/10.1016/j.jcf.2015.10.014 32- Pena Amaya P, Haim M, Fernández S, Di Gregorio S, Teper A, Vázquez M et al. Molecular Epidemiology of Methicillin-Resistant Staphylococcus aureus in Cystic Fibrosis Patients from Argentina. Microbial Drug Resistance. 2017;24(5):613-620. 33Klaudia Cios, Bevin Cohen, Lynne M. Quittell, Jianfang Liu, Elaine L. Larson. Impact of colonizing organism in the respiratory tract on the incidence, duration, and time between subsequent hospitalizations among patients with cystic fibrosis. American Journal of Infection Control 000 (2019) 1 -5 https://doi.org/10.1016/j.ajic.2018.12 .021 34- Jennings MT, et al, Risk factors for persistent methicillin-resistant Staphylococcus aureus infection in cystic fibrosis, J Cyst Fibros (2017), http://dx.doi.org/10.1016/j.jcf.2017.04.010 35- Keli Cristine Reiter, Alice Beatriz Mombach Pinheiro Machado, Ana Lúcia Peixoto de Freitas, Afonso Luís Barth. High prevalence of methicillin-resistant Staphylococcus aureus with SCC mec type III in cystic fibrosis patients in southern, Brazil. Revista da Sociedade Brasileira de Medicina Tropical 43(4):377-381, jul-ago, 2010. 36- Bittencourt PH, Pimentel CSS, Bonfim BS, Marostica PJ, Souza EL. Incidence and treatment of methicillin-resistant S. aureus infection in cystic fibrosis patients: a cohort study. braz j infect dis $2017 ; 21$ (1):107-111 37- Clement L. Ren, et al. Presence of Methicillin Resistant Staphylococcus aureus in Respiratory Cultures From Cystic Fibrosis 
Patients Is Associated With Lower Lung Function. Pediatric Pulmonology 42:513-518 (2007) 38Michelle E. Wood, Laura J. Sherrard, Kay A. Ramsay, Stephanie T. Yerkovich, David W. Reid, Timothy J. Kidd, Scott C. Bell. Methicillin-resistant Staphylococcus aureus acquisition in healthcare workers with cystic fibrosis: a retrospective cross-sectional study. BMC Pulmonary Medicine (2016) 16:78. DOI 10.1186/s12890-016-0243-z 39- Nobandegani NM, Mahmoudi S, Pourakbari B, Sadeghi RH, Sani MN, Farahmand F, Motamed F, Rafsanjani RN, Mamishi S, Antimicrobial susceptibility of microorganisms isolated from sputum culture of patients with cystic fibrosis: Methicillin-resistant Staphylococcus aureus as a serious concern, Microbial Pathogenesis (2016), doi: 10.1016/j.micpath.2016.09.015 40- T. Goolam Mahomed, M. M. Kock, R. Masekela, E. Hoosien, M. M. Ehlers. Genetic relatedness of Staphylococcus aureus isolates obtained from cystic fbrosis patients at a tertiary academic hospital in Pretoria, South Africa. Scientific Reports (2018) 8:12222. DOI:10.1038/s41598-018-30725-x 41- Govan JRW. Infection control in cystic fibrosis: methicillin-resistant Staphylococcus aureus, Pseudomonas aeruginosa and the Burkholderia cepacia complex. J R Soc Med 2000; 93(Suppl. 38):40-45 42- Hiramatsu K, Cui L, Kuroda $\mathrm{M}$, Ito T. The emergence and evolution of methicillin-resistant Staphylococcus aureus. Trends in microbiology. 2001 Oct 1;9 (10):486-93. 43- Jennings MT, Riekert KA, Boyle MP. Update on Key Emerging Challenges in Cystic Fibrosis. Med Princ Pract 2014;23:393-402 DOI: 10.1159/000357646 44- Ronald L. Gibson, Jane L. Burns, Bonnie W. Ramsey. Pathophysiology and Management of Pulmonary Infections in Cystic Fibrosis. AMERICAN JOURNAL OF RESPIRATORY AND CRITICAL CARE MEDICINE 2003; vol 168.

\section{Figure Legends}

- Figure 1: flow chart for study selection process.

- Figure 2: pooled prevalence of prevalence of MRSA colonization in CF patients.

- Figure 3: Funnel plot for publication bias assessment.

- Figure 4 A-B: Meta regression scatters plots for correlation between prevalence and sample size or publication year.

\section{Tables}

Due to technical limitations, tables are only available as a download in the supplemental files section.

\section{Figures}




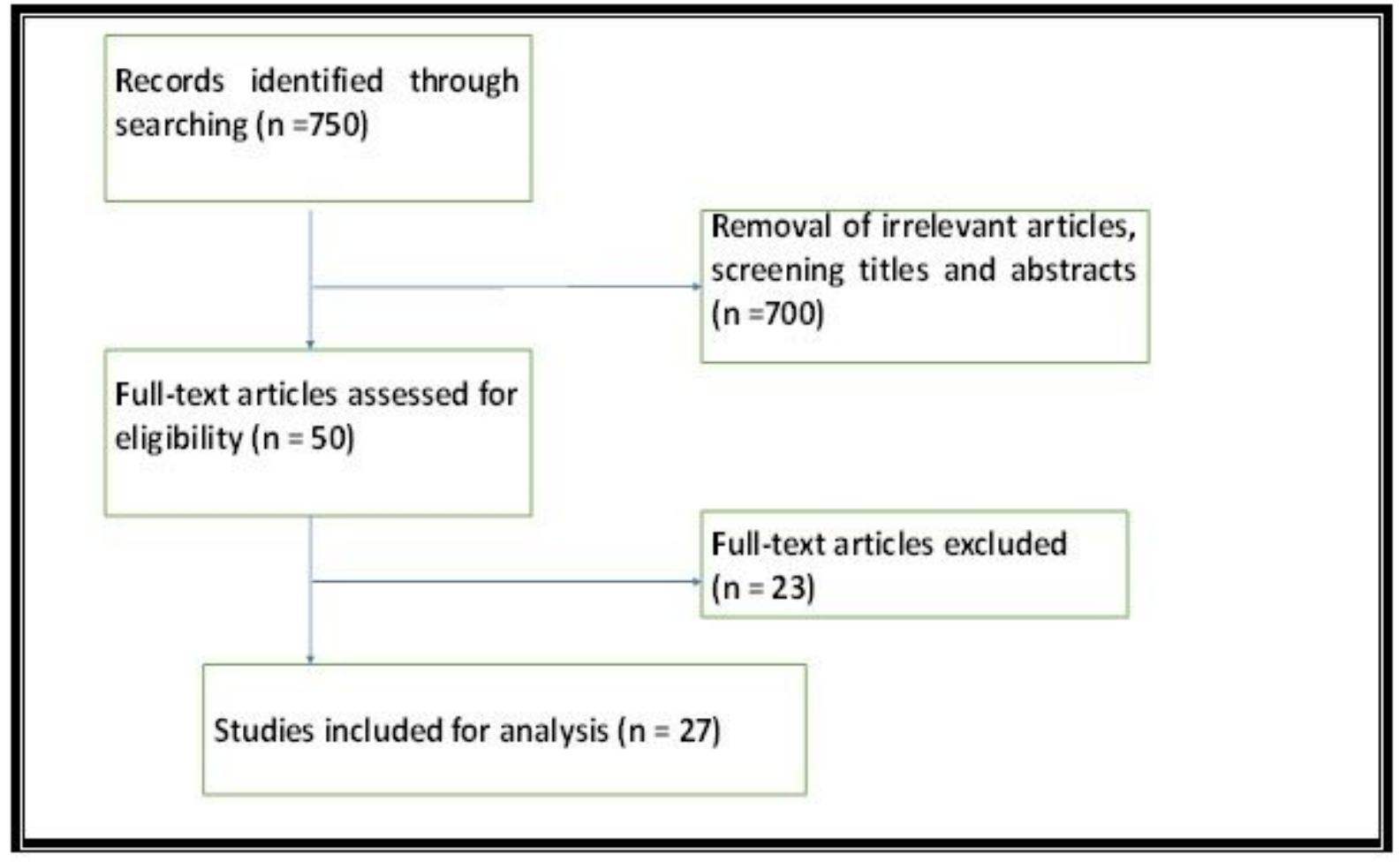

Figure 1

Figure 1: flow chart for study selection process. 


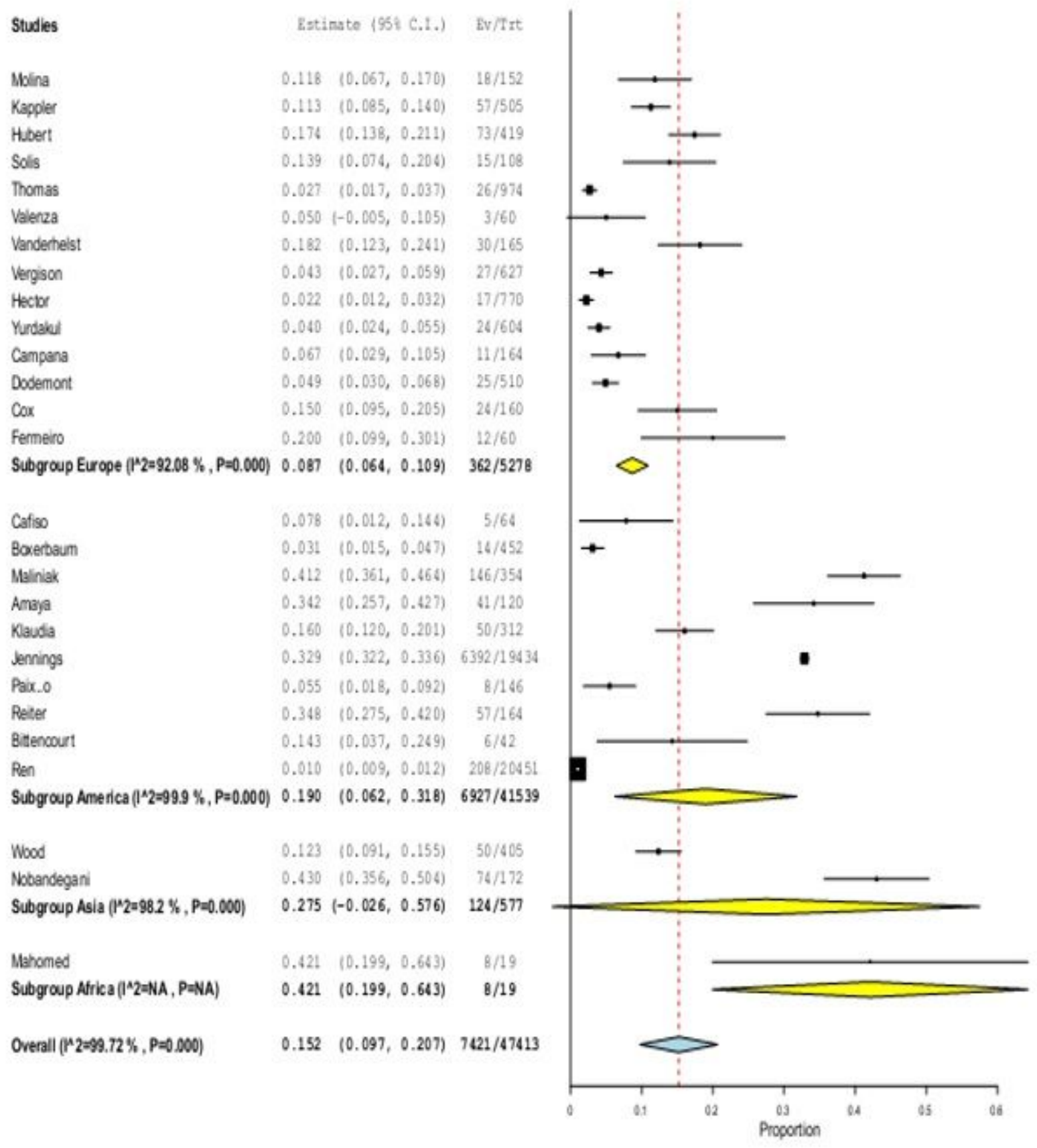

Figure 2

Figure 2: pooled prevalence of prevalence of MRSA colonization in CF patients. 


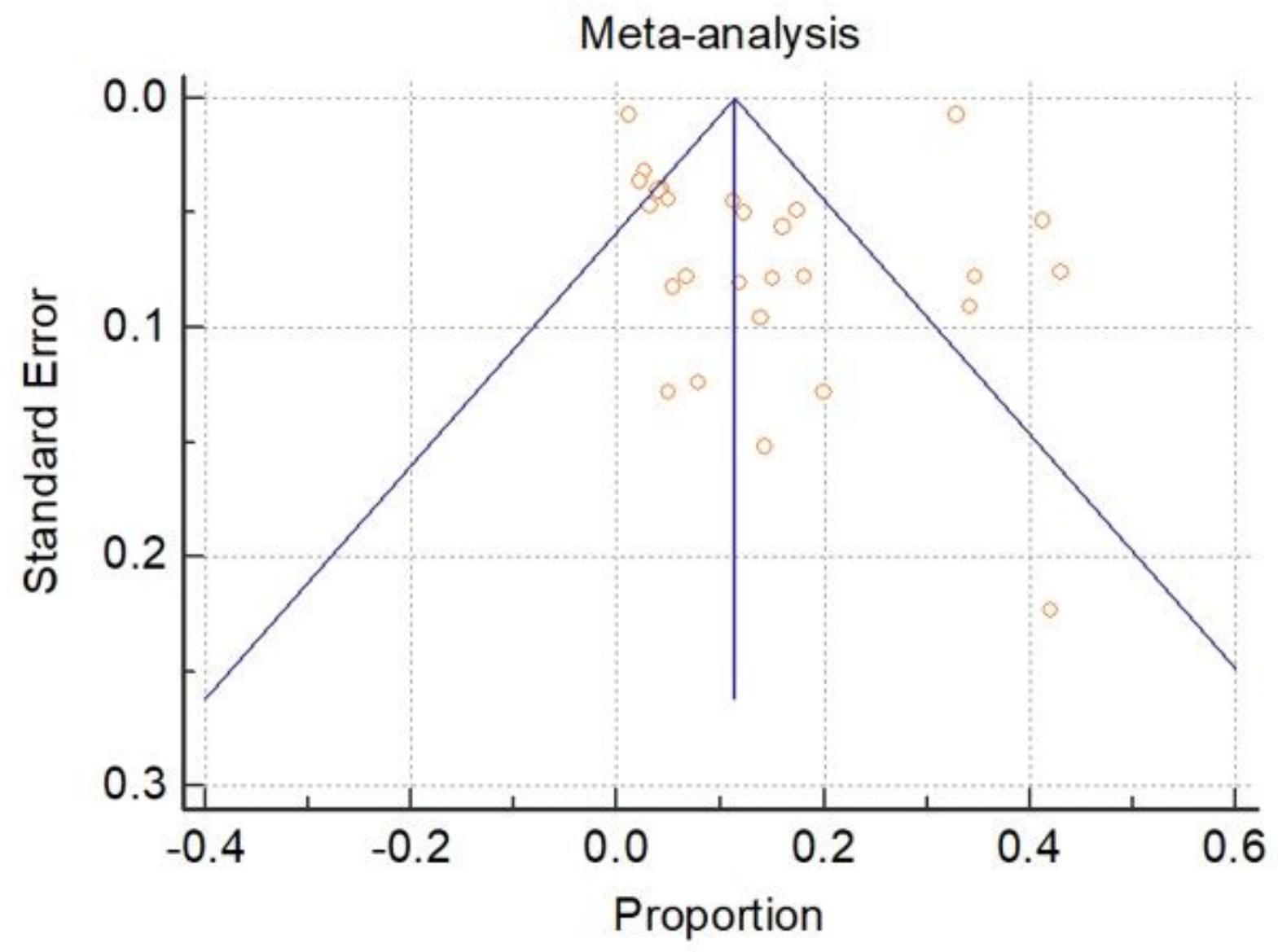

Figure 3

Figure 3: Funnel plot for publication bias assessment. 


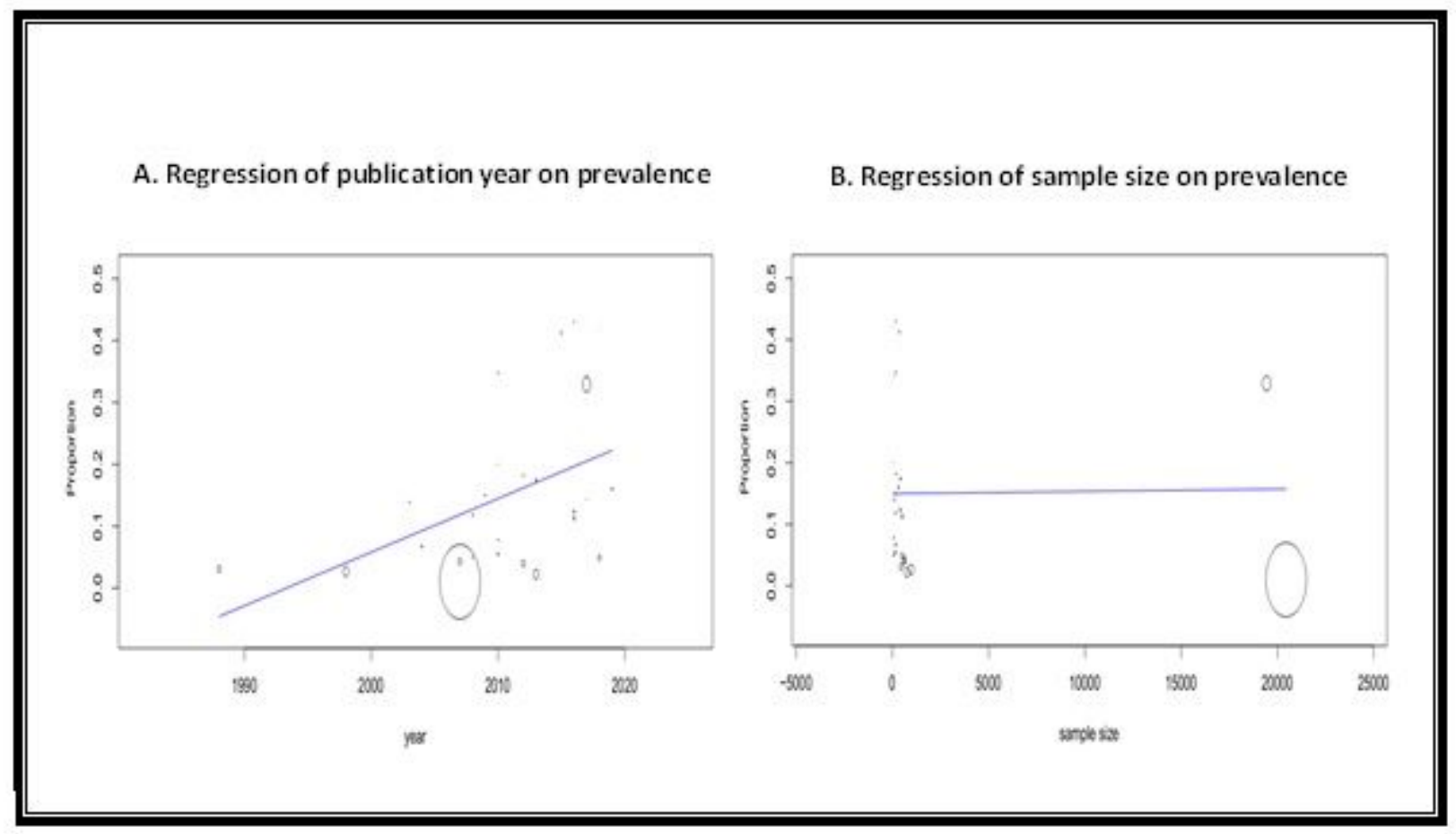

Figure 4

Figure 4 A-B: Meta regression scatters plots for correlation between prevalence and sample size or publication year.

\section{Supplementary Files}

This is a list of supplementary files associated with this preprint. Click to download.

- Table1.jpg

- Table2.jpg 